\title{
Correction to: Phenotypic Analysis of Fruit Crops Water Stress Using Infrared Thermal Imaging
}

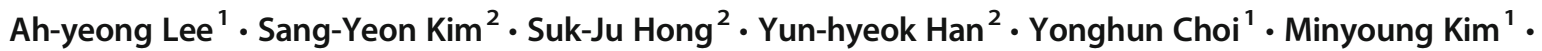 \\ Seok Kyu Yun ${ }^{3}$. Ghiseok Kim ${ }^{2,4}$
}

Published online: 3 September 2019

(C) The Korean Society for Agricultural Machinery 2019

Correction to: Journal of Biosystems Engineering (2019) 44:87-94 https://doi.org/10.1007/s42853-019-00020-2

Due to an unfortunate mistake, the author name "Younghun Choi" has been misspelt. It should be read as "Yonghun Choi."

Ghiseok Kim

ghiseok@snu.ac.kr

Ah-yeong Lee lay117@korea.kr

Sang-Yeon Kim yskra@snu.ac.kr

Suk-Ju Hong hsj5596@snu.ac.kr

Yun-hyeok Han redstar316@snu.ac.kr

Yonghun Choi yhchoi82@korea.kr

Minyoung Kim Mykim75@korea.kr
Seok Kyu Yun

sky0611@korea.kr

1 Rural Development Administration, National Institute of Agricultural Sciences, Wanju, Jeollabuk-do 54875, Republic of Korea

2 Department of Biosystems and Biomaterials Science and Engineering, Seoul National University, 1 Gwanak-ro, Gwanak-gu, Seoul 08826, Republic of Korea

3 Fruit Research Division, National Institute of Horticultural \& Herbal Science, Wanju, Republic of Korea

4 Research Institute of Agriculture and Life Sciences, Seoul National University, 1 Gwanak-ro, Gwanak-gu, Seoul 08826, Republic of Korea 